\title{
Lessons We have Learnt from the Fight Against Tubrculosis During the Past 100 Years
}

\author{
Tadao Shimao, Director \\ Research Institute of Tuberculosis, Japan Anti-Tuberculosis Association
}

\section{Introduction}

This year, 1982, is the centenary of the discovery of tubercle bacilli by Robert Koch. Tuberculosis was highly prevalent in European countries by that time; the mortality rate from tuberculosis was approximately 300 per 100,000 in Germany, and the people were afraid of tuberculosis. Koch's discovery was indeed a great scientific achievement as the causing agent of such a dreadful disease was first discovered.

His work was perfect: 1) he discovered the bacilli in tuberculous lesions and such bacilli were not discovered in lesions of other diseases, 2) he could succeed to culture the bacilli successively, 3) he could produce tuberculosis in animals inoculated with the cultured bacilli, and 4) he discovered the same bacilli in tuberculous lesions of animals.

He was honored in 1905 the Nobel Prize for this excellent work. His work was no doubt an excellent original research, but we have to recognize that several advances in related science supported the discovery of tubercle bacilli by R. Koch. Among them, we have to point out the followings particularly: 1) the use of condenser and oil immersion by Abbe which improved markedly the resolution power of microscope, and 2) advances in dye industry in Germany which made possible the staining of bacilli easier.

Time Interval from Discovery of Tubercle Bacilli to Development of Tuberculosis Control Measures.
The discovery of causing agent is a first step to develop preventive, diagnostic and curative measures, but it took a certain period of time to accomplish them. The followings are the interval from the discovery of tubercle bacilli to the development of important tuberculosis control measures:

Preventive measures.

1908 Culture of BCG was initiated. (Calmette and Guerin)

1921 First oral use of BCG vaccine to infants. (Weille-Alle)

Diagnostic measures.

1890 Old Tuberculin was prepared. (Koch)

1908 Tuberculin percutaneous test was used to identify tuberculosis infection. (Pirquet)

$1895 \mathrm{X}$-ray was discovered. (Roentgen)

1936 Discovery of radiophotography. (Koga and de Abreu)

Chemotherapy.

1944 Streptomycin was discovered. (Waksman)

1966 Rifampicin was produced. (Lepetit and Ciba-Geigy)

1970s Short-course sterilizing chemotherapy was developed. (Fox and BMRC)

\section{Advances in Tuberculosis Immunology}

Several phenomena suggesting the presence of tuberculosis immunity were already recognized by several research workers. Koch himself noticed Koch's phenomena; rapid and intensive local reactions and slight regional glandular reaction to re-challenge in contrast to slow but moderate local 
as well as regional glandular reactions to primary infection of bacilli.

Pirquet (1908) introduced the tuberculin skin reaction, and positive reaction to tuberculin was found to indicate the presence of tuberculosis infection, as not only tuberculosis patients but also those with healed primary complex alone showed positive reaction to tuberculin.

The effectiveness of BCG vaccination was a good proof of tuberculosis immunity. Heimbeck found the clear difference in the incidence of tuberculosis among nurses starting to work in tuberculosis sanatorium according to the initial reaction to tuberculin. Among initially non-reactors, the incidence rate of tuberculosis was $27.0 \%$ (50 out of 185), while it was $1.3 \%$ (2 out of 152) among initially positive reactors to tuberculin. All these facts suggested the presence of tuberculosis immunity, but its real mechanism was unknown until recently.

It was only during past two decades that the mechanisms of tuberculosis immunity was elucidated. It was found that the sensitized T-lymphocyte was playing a key role, and it had a capacity to identify tubercle bacilli or other related substances such as tuberculin, then started to multiply actively and liberate lymphokines. The latter had several biological activities such as MAF, MIF and SRF. Macrophages activated by lymphokines could cope with tubercle bacilli much better than normal macrophages.

Tuberculosis is a good model to understand the cell-mediated immunity, and tubercle bacilli are important tools in the immunological research.

\section{Tuberculosis Case-finding}

Mass Miniature Radiography (MMR), an active approach to the people, was used as a spear-head to organize the national tuberculosis control programme, and it played an important role to promote the community participation to the programme.

It was found, however, that the majority of new tuberculosis patients, especially smear positive cases, were detected by symptomatic visit to health facilities, and only a small portion of new cases were detected by MMR even in countries where MMR is still actively carried out. The fact could be explained first by the both quantitative and qualitative limits in MMR and second by the: nature of tuberculosis disease itself.

If MMR is carried out repeatedly on the same population groups, those who were examined and not examined were tended to be fixed, and it is practically very difficult to examine those who refuse to join in spite of intensive invitation. To keep the quality of MMR is also very difficult when the MMR is carried out nation-wide.

Another important fact is that tuberculosis is: not so asymptomatic and not so slowly progressing as it was believed to be. Pulmonary tuberculosis develops from cavity by step-like progression and during and soon after the progression patients complain signs of acute respiratory infections as slight fever, tiredness, malaise, cough and sputum. All these symptoms are alike those of common cold, but in the case of common cold, such symptoms may disappear within a few days or a week, while in the case of tuberculosis progression, they persist longer, and patients were motivated to visit health facilities. The speed of progression of the disease was also important. By the retrospective review of proevious MMR, approximately $50 \%$ of new bacillary cases showed normal or only healed. findings on their chest X-ray within one year. Such so-called rapid cases were hardly discovered by repeated MMR.

Considering all these facts, most important in early detection of tuberculosis patients is symptomatic or passive case-finding, and MMR or active case-finding has only a certain limited role even in technically advanced countries. Delay in casefinding is divided into patients's delay and doctor's delay. Intensive health education programme should be made to the general public to promote the early visit to health facilities when they had persisting respiratory symptoms. Staff 
in health facilities should be instructed to pick up those who complained symptoms suggesting tuberculosis from visitors and make appropriate examinations for such cases.

\section{Tuberculosis Chemotherapy}

After the discovery of streptomycin by Waksman in 1944, chemotherapy for tuberculosis showed peculiar advances. Streptomycin showed an excellent effect to laryngeal and intestinal tuberculosis, it stopped laryngeal pain and diarrhea which were very difficult to manage by other methods, and patient's general conditions were improved markedly within a short period of time after starting the use of streptomycin. The drug, however, became not effective to pulmonary cavity, as bacilli in cavity became resistant to streptomycin. The mechanisms of emergence of drug resistance to anti-tuberculous drugs is explained by the incidence of drug resistant mutant, a probability of which is approximately $10^{-5}$ to $10^{-6}$. As the number of tubercle bacilli in cavity is on a level of $10^{8}$, if a drug is used alone, some of bacilli in a cavity became resistant to the drug sooner or later, which can multiply under the presence of the drug while sensitive ones are killed. Soon after the discovery of streptomycin, another new drug, PAS was discovered by Lehman. The use of combined regimen of SM and PAS was an excellent idea to overcome the emergence of drug resistance of tubercle bacilli. Isoniazid was found to be effective in 1952 for tuberculosis, and since then, the triple combination of INH, SM and PAS had been used as the standard regimen of chemotherapy for tuberculosis for so many years.

Introduction of the controlled trial was also an important progress in chemotherapy for tuberculosis. This enabled us to compare objectively the effectiveness of various drug regimens, and moreover, the effectiveness of different methods of treatment, such as hospital versus home treatment under the same regimen of chemotherapy: This methodology has been applied in various other clinical trials.

The concept of case-holding was introduced first in tuberculosis, as patients have to take drugs regularly for very long period of time, and the importance of patient's compliance was recognized in the treatment of tuberculosis. As the defaulter rate during treatment was so high in many developing countries, an idea of supervised treatment was introduced. The concept of case-holding is now applied to many other chronic disease control.

The method of administration of antituberculous drugs is also different from other antibiotics, in which drugs should be taken at regular interval of 6 to 8 hours. In the case of antituberculous drugs, the intermittent use was very often tried and it was found that the daily use of ordinary dose is equally effective to the high dose intermittency. A single administration of daily dose is equal or superior to divided use of the same dose in its effectiveness and the incidence of side-effects.

One more important fact which was recognized by the study of tuberculosis chemotherapy was the role played by the host defense mechanisms. The short-course chemotherapy using INH, RFP and other potent drugs is quite effective, and the relapse after the completion of prescribed regimen was very rare. Tubercle bacilli were seemd to be killed in vivo. The use of steroid, however, causes relapse even after the completion of treatment in animals, and sterilization of tubercle bacilli was difficult in the case of thymectomized mouse. All these facts show the importance of host defense mechanisms in suppressing the regrowth of tubercle bacilli thus preventing the relapse.

\section{Epidemiology of Tuberculosis}

As a study subject for epidemiology, tuberculosis is one of the most important ones, as only a part of the population get infected and only a part of them develop clinical tuberculosis, and factors on the side of causing agent, host and surrounding conditions complicatedly relate to the incidence of 
infection, breakdown and development of the disease.

Mortality from tuberculosis has long been used as an epidemiological index to assess the magnitude of tuberculosis problem. Due to rapid changes in the pattern of tuberculosis death especially pulmonary tuberculosis death with cor pulmonale and negative bacilli in sputum and increase of non-tuberculosis death relating to tuberculosis, its reliability as, an epidemiological index was diminished in technically advanced countries.

Incidence and prevalence of tuberculosis were very difficult to compare internationally, as the definition of the case of tuberculosis was different from country to country, and the figures were very much influenced by the programme coverage.

There was an urgent need to develop a new reliable epidemiological index to assess the magnitude of tuberculosis problems. Tuberculosis Surveillance Research Unit(TSRU) succeeded to introduce the concept of annual risk of tuberculosis infection. This index could be obtained by making tuberculin survey on appropriately selected random population by using proper technique either on the same group at a certain interval or on the different age groups. The annual risk of tuberculosis infection was found to be a sole reliable index to measure the magnitude of tuberculosis problem in the community. Its application, however, is limited in countries where the coverage of BCG vaccination is high in children.

Experiences in West European and North American countries indicate that tuberculosis could be reduced without any tuberculosis control measures but by improving the living standard. Better housing prevents the infection and better nutrition prevents the breakdown and development of the disease. The annual decline of tuberculosis by improving the living standard was approximately 4 to $5 \%$. The decline of tuberculosis in technically advanced countries during the past 30 decades could be explained by the combination of the implementation of tuberculosis control programme and the improvement of living standard, and the overall annual decline was 10 to $15 \%$. Thus, tuberculosiscontrol programme contributed to the annual. decline of 6 to $10 \%$.

In reducing tuberculosis problem, the appropriately implemented tuberculosis case-finding and treatment progrmmes played a major role, as the chain of transmission of tubercle bacilli could be cut down by time. The impact of BCG vaccination was not so big as it was expected, as it prevents. tuberculosis mainly in children, which are noninfectious in the majority of cases.

\section{Future Direction of Tuberculosis Research}

Through the application of advanced technology, tuberculosis has been brought under control in the technically advanced countries. However, tuberculosis still remains as one of the major public health problems in the majority of developing. countries.

WHO set a target "Health for All by the Year 2000 " and try to reach the target through primary health care approach. Health researches which are composed of biomedical health service and behavioural researches, should be directed to accomplish the target by distributing the benefits of advances in biomedical researches to every people in the most peripheral part of the country. As. biomedical research was already well developed in tuberculosis, emphasis of research should be given. to health service and behavioural researches especially in developing countries, though several problem should be solved in biomedical research in. the future.

As biomedical researches, studies on the mechanisms of endogenous reactivation and relapse after chemotherapy might be one of the most important, as the acceleration of decline of tuberculosis is: expected by elucidating its mechanisms and finding: out measures to prevent them.

In the field of chemotherapy, studies on the application of short-course chemotherapy under field condition in developing countries are to bedone. Studies on the short-course regimen of che-- 
motherapy for smear negative cases and extrapulmonary tuberculosis are also needed.

Although the annual risk of tuberculosis infection was found to be a sole reliable index to measure the magnitude of tuberculosis problem, the coverage of BCG vaccination in children is often very high at present. The role of tuberculosis prevalence survey should be investigated under such conditions.

Operational and sociological investigations are very important, and the following topics are suggested as the top priority:

How to overcome difficulties encountred during the integration of tuberculosis control programme into primary health care system.

How to intensify the case-finding and case-hold ing activities.

How far we can dencentralize bacteriological examinations. Merit and demerit of decentralization should be investigated under local conditions existing in developing countries.

How to implement technical supervision for peripheral workers.

During the past 100 years since the discovery of tubercle bacilli by $\mathrm{R}$. Koch, tuberculosis has been brought under control in technically advanced countries through the application of advanced scientific achievements. Tuberculosis, however, still remains as one of the most important health problems in many developing countries of the world. The occasion of the centenary of Koch's discovery should be used not only to review and praise the achievements in the past, but also to consider prospectively the future direction of tuberculosis research and control programme, with special emphasis on developing countries, based on lessons we have learnt during the past 100 years. These experiences could be applied also in the fight against other diseases. Tuberculosis is indeed a model in various aspects. 